University of New Hampshire

University of New Hampshire Scholars' Repository

Space Science Center

Institute for the Study of Earth, Oceans, and

Space (EOS)

2001

\title{
COMPTEL observations of the blazars 3C 454.3 and CTA 102
}

\author{
S Zhang \\ Max-Planck-Institut für extraterrestriche Physik \\ W Collmar \\ Max-Planck-Institut für extraterrestriche Physik \\ V Schonfelder \\ Max-Planck-Institut für extraterrestrische Physik \\ H Bloemen \\ Space Research Organization of the Netherlands \\ W Hermsen \\ Space Research Organization of the Netherlands (SRON)
}

See next page for additional authors

Follow this and additional works at: https://scholars.unh.edu/ssc

Part of the Astrophysics and Astronomy Commons

\section{Recommended Citation}

COMPTEL observations of the blazars 3C 454.3 and CTA 102 Zhang, S. and Collmar, W. and Schönfelder, V. and Bloemen, $\mathrm{H}$. and Hermsen, W. and McConnell, M. and Bennett, K. and Williams, O. R., AIP Conference Proceedings, 587, 343-347 (2001), DOI:http://dx.doi.org/10.1063/1.1419427

This Conference Proceeding is brought to you for free and open access by the Institute for the Study of Earth, Oceans, and Space (EOS) at University of New Hampshire Scholars' Repository. It has been accepted for inclusion in Space Science Center by an authorized administrator of University of New Hampshire Scholars' Repository. For more information, please contact Scholarly.Communication@unh.edu. 


\section{Authors}

S Zhang, W Collmar, V Schonfelder, H Bloemen, W Hermsen, Mark L. McConnell, K Bennett, and O R. Williams 


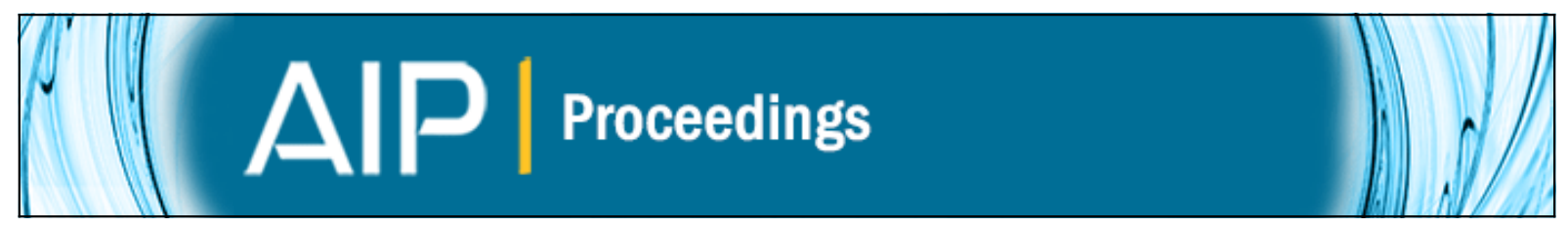

\section{COMPTEL observations of the blazars 3C 454.3 and CTA 102}

S. Zhang, W. Collmar, V. Schönfelder, H. Bloemen, W. Hermsen, M. McConnell, K. Bennett, and O. R. Williams

Citation: AIP Conference Proceedings 587, 343 (2001); doi: 10.1063/1.1419427

View online: http://dx.doi.org/10.1063/1.1419427

View Table of Contents: http://scitation.aip.org/content/aip/proceeding/aipcp/587?ver=pdfcov

Published by the AIP Publishing

\section{Articles you may be interested in}

COMPTEL observations of the Virgo blazars 3C 273 and 3C 279

AIP Conf. Proc. 587, 271 (2001); 10.1063/1.1419412

Multifrequency observations of the Virgo blazars 3C 273 and 3C 279 in CGRO cycle 8

AIP Conf. Proc. 510, 303 (2000); 10.1063/1.1303222

VLBA monitoring of three gamma-ray bright blazars: AO 0235+164, 1633+382 (4C 38.41) \& 2230+114 (CTA 102)

AIP Conf. Proc. 410, 1437 (1997); 10.1063/1.54073

Variability time scales in the gamma-ray blazars using structure function analysis

AIP Conf. Proc. 410, 1361 (1997); 10.1063/1.53947

Further COMPTEL observations of the region around GRO J1753 +57: are there several MeV sources present? AIP Conf. Proc. 410, 1243 (1997); 10.1063/1.53935 


\title{
COMPTEL Observations of the Blazars 3C 454.3 and CTA 102
}

\author{
S. Zhang ${ }^{1,5}$, W. Collmar ${ }^{1}$, V. Schönfelder ${ }^{1}$, H. Bloemen ${ }^{2}$, \\ W. Hermsen ${ }^{2}$, M. McConnell ${ }^{3}$, K.Bennett ${ }^{4}$, O.R. Williams ${ }^{4}$ \\ ${ }^{1}$ Max-Planck-Institut für extraterrestrische Physik, Garching, Germany \\ ${ }^{2}$ Space Research Organization Netherlands, Utrecht, The Netherlands \\ ${ }^{3}$ Space Science Center, University of New Hampshire, Durham, USA \\ ${ }^{4}$ Astrophysics Division, ESTEC, Noordwijk, The Netherlands \\ ${ }^{5}$ High Energy Astrophysics Lab, IHEP, P.O.Box 918-3, Beijing, China
}

\begin{abstract}
We have analyzed the two blazars of 3C 454.3 and CTA 102 using all available COMPTEL data from 1991 to 1999 . In the 10-30 MeV band, emission from the general direction of the sources is found at the $4 \sigma$-level, being consistent with contributions from both sources. Below $10 \mathrm{MeV}$ only $3 \mathrm{C} 454.3$ is significantly detected, with the strongest evidence $(5.6 \sigma)$ in the $3-10 \mathrm{MeV}$ band. Significant flux variability is not observed for both sources, while a low emission is seen most of the years in the 3-10 MeV light curve for $3 \mathrm{C} 454.3$. Its time-averaged $\mathrm{MeV}$ spectrum suggests a power maximum between 3 to $10 \mathrm{MeV}$.
\end{abstract}

\section{INTRODUCTION}

Gamma-ray emission from the two blazars 3C 454.3 and CTA 102, located $\sim 7^{\circ}$ apart on the sky, was discovered by EGRET during the early Compton Gamma-Ray Observatory (CGRO) mission [1,2]. COMPTEL measurements at $\mathrm{MeV}$ energies of the same time periods, reported earlier by Blom et al. [3], show indications that both sources are weakly detected in the COMPTEL uppermost (10-30 MeV) band. If combined with EGRET, the trend for a spectral flattening at $\mathrm{MeV}$ energies becomes visible. At even lower energies (hard X-rays) OSSE detected variability of both sources in 1994 [4].

In this paper, we present the results of the COMPTEL observations on both blazars during the whole CGRO mission. The data have been consistently analyzed for both blazars and compared to the results obtained early in the mission, published by Blom et al. [3]. A more detailed presentation of the analysis results will be given by Zhang et al. [5].

CP587, GAMMA 2001: Gamma-Ray Astrophysics 2001, edited by S. Ritz et al. (C) 2001 American Institute of Physics 0-7354-0027-X/01/\$18.00 

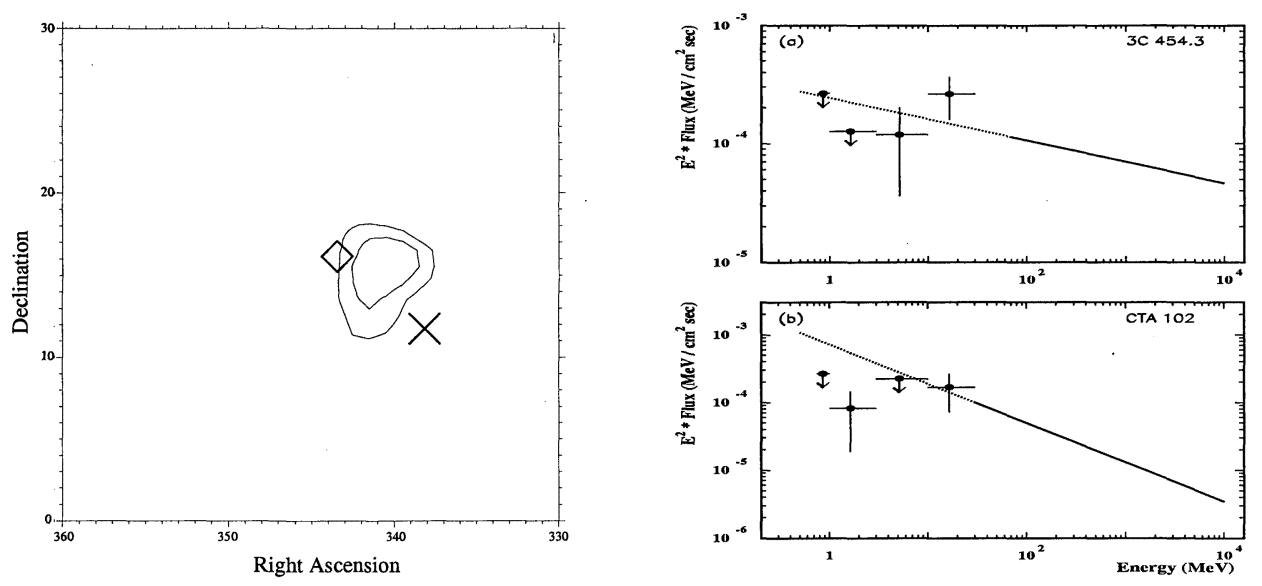

FIGURE 1. Left: COMPTEL 10-30 MeV map for 3C $454.3(\diamond)$ and CTA $102(\times)$ from Phase 1 observations in 1992. The contour lines start at a detection significance level of $3 \sigma$ with steps of $0.5 \sigma$. Right: Combined COMPTEL/EGRET spectra for 3C 454.3 (a) and CTA 102 (b). The filled circles represent the COMPTEL spectra of Phase 1 (VPs 19.0, 26.0, 28.0 and 37.0), the solid lines the EGRET spectra of VP $19[1,2]$ and the dotted lines their extrapolations toward lower energies. The error bars are $1 \sigma$ and the upper limits $2 \sigma$.

\section{DATA AND ANALYSIS METHOD}

The imaging Compton Telescope COMPTEL - one of four experiments aboard CGRO - was sensitive to $\gamma$-rays in the energy range $0.75-30 \mathrm{MeV}$ [6]. During its whole mission $(1991-2000)$, the sources were in 22 CGRO viewing period (VPs) within $40^{\circ}$ of the COMPTEL pointing direction. These VPs were selected in our analyses.

The analyses were carried out by using the standard COMPTEL maximumlikelihood analysis procedure including a filtering technique for background gener-

ation. Point spread functions of the instrument which assume an $\mathrm{E}^{-2}$ power law shape for the input spectrum were applied in our analyses.

\section{RESULTS}

\section{Phase 1}

For CGRO Phase 1 (April '91 to November '92) our results are similar to those reported by Blom et al. [3]. There is evidence for emission from the general direction of 3C 454.3 and CTA 102 in the 10-30 MeV band with a detection significance of $3.9 \sigma$, which is consistent with contributions from both sources (Figure 1). At lower 

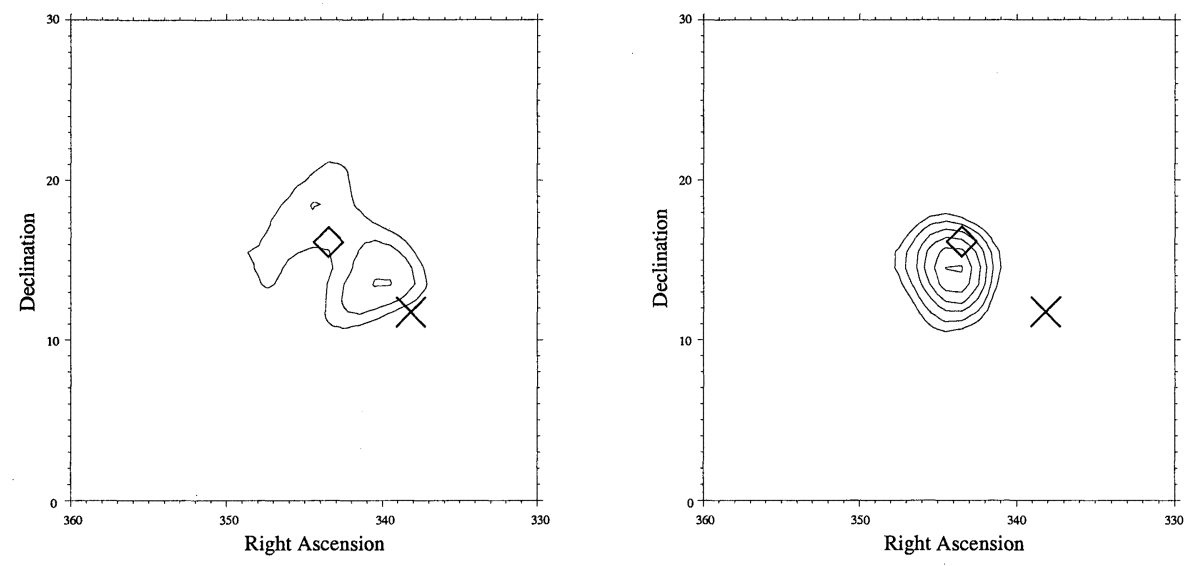

FIGURE 2. COMPTEL 10-30 MeV map (left) and 3-10 MeV map (right) for 3C $454.3(\diamond)$ and CTA $102(x)$ from 8 years of observations. The contour lines start at a detection significance level of $3 \sigma$ with steps of $0.5 \sigma$.

energies, only marginal detections and upper limits are obtained. The combined COMPTEL/EGRET spectra indicate spectral turnovers at MeV energies for both sources (Figure 1).

\section{Phases 1-8}

No significant $(>3 \sigma)$ detection is found for either 3C 454.3 or CTA 102 in any individual COMPTEL observation during the whole CGRO mission in the four standard energy ranges. However, combining all the COMPTEL data provides evidence for 3C 454.3 at energies above $1 \mathrm{MeV}$ and for CTA 102 at energies above $10 \mathrm{MeV}$. The 10-30 MeV skymap (Figure 2) shows that a $4 \sigma$-excess is located just between 3C 454.3 and CTA 102, again consistent with emission from both. No signal can be derived from CTA 102 in the $3-10 \mathrm{MeV}$ band, while an obvious excess is detected near 3C 454.3 at a significance level of $\sim 5.6 \sigma$ (see Figure 2). 3C 454.3 is located at the $4.5 \sigma$-detection contour and is within the $3 \sigma$ error location contour. Since 3C 454.3 is the only known $\gamma$-ray source in the COMPTEL error box, we attribute this significant excess to emission from 3C 454.3 .

A search for flux variability has been carried out in all individual observations on 3C 454.3 and CTA 102 in the four standard COMPTEL energy bands. All light curves are consistent with a constant flux for both sources over a period of about 8 years. While only marginal hints or upper limits are found along the CGRO mission for CTA 102, 3C 454.3 seem to be always emitting at somehow a low level 

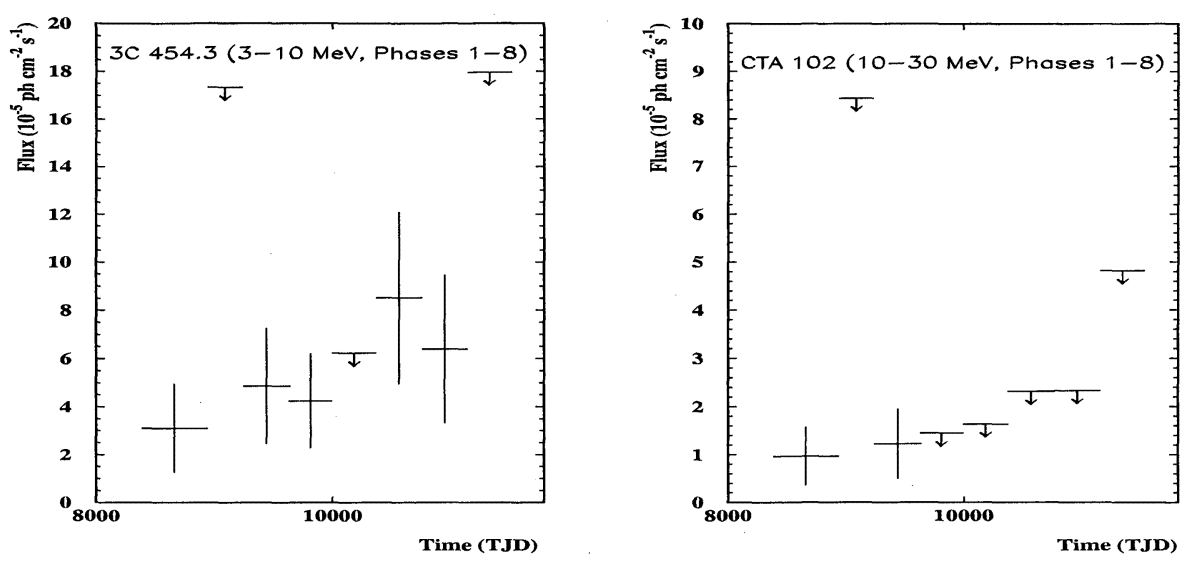

FIGURE 3. The 3-10 MeV light curves for 3C 454.3 (left) and the $10-30 \mathrm{MeV}$ light curve for CTA 102 (right) with each bin averaged over one CGRO Phase which typically covers a time period of 1 year. The high upper limits show times of low source exposures. The error bars are $1 \sigma$ and the upper limits $2 \sigma$.

in the $3-10 \mathrm{MeV}$ band (Figure 3). This is consistent with the significant detection of $3 \mathrm{C} 454.3$ in the sum of the $3-10 \mathrm{MeV}$ data. The $10-30 \mathrm{MeV}$ light curve of CTA 102 (Figure 3 ) shows mostly upper limits, indicating the source was weak along the CGRO mission.

Figure 4 shows the COMPTEL spectra averaged over a time period between April 1991 and November 1999 for both sources. The spectrum of 3C 454.3 (Figure 4) suggests a power maximum (at least with respect to $\mathrm{MeV}$ energies) in the 3-10 $\mathrm{MeV}$ band. No conclusion can be derived from the spectrum of CTA 102 because only in the $10-30 \mathrm{MeV}$ band a weak detection is obtained (Figure 4).

\section{DISCUSSION AND SUMMARY}

Our analyses of the data of the early COMPTEL observations of the $\gamma$-loud flat-spectrum radio quasars 3C 454.3 and CTA 102 provide similar results with respect to the weak source detections and spectra as reported by Blom et al. [3]. The consistent analysis of all available COMPTEL data, covering 8 years, reveals some new features for these two blazars. The most important one is the significant detection of $3 \mathrm{C} 454.3$ in the $3-10 \mathrm{MeV}$ band. Furthermore, $3 \mathrm{C} 454.3$ seems to be a emitter always detectable in the $3-10 \mathrm{MeV}$ band, where it appears to reach its power maximum in the time-averaged spectrum. It resembles $3 \mathrm{C} 273$ which is a rather stable $\mathrm{MeV}$ emitter with a power maximum in the $3-10 \mathrm{MeV}$ band [10], if the upper limits in the 3-10 MeV light curve are detections of $3 \mathrm{C} 454.3$ near the threshold of COMPTEL. Therefore, 3C 454.3 might internally be similar to 

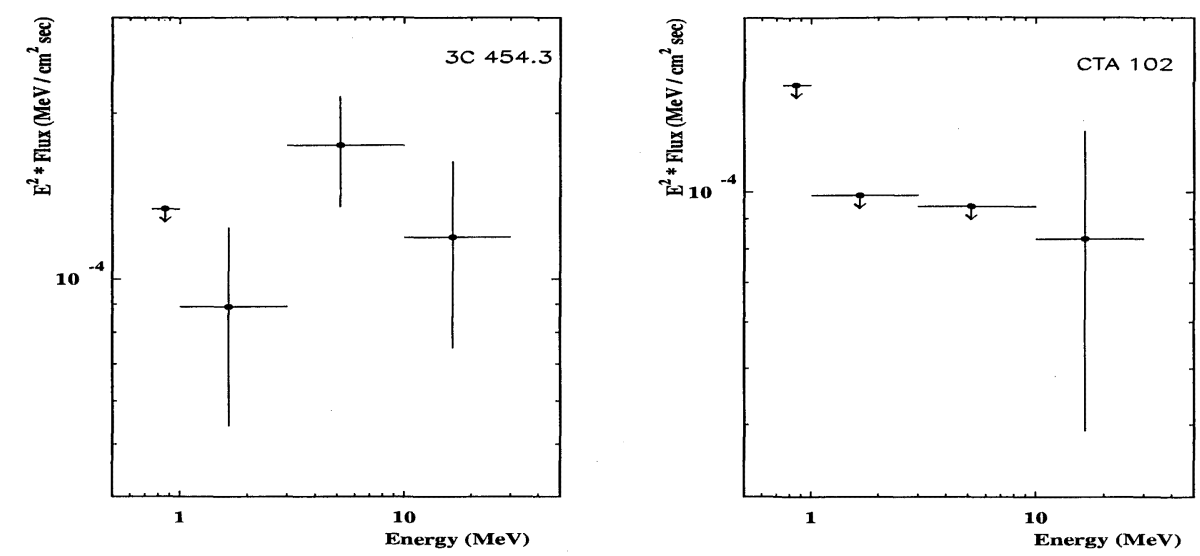

FIGURE 4. COMPTEL spectra for 3C 454.3 (left) and CTA 102 (right) averaged over observations in Phases 1-8. The error bars are $1 \sigma$ and the upper limits $2 \sigma$.

3C 273 however at a lower flux level since we just see the 'tip of the iceberg' with COMPTEL. Compared to 3C 454.3, the MeV emission from CTA 102 is weak and the detection significance marginal. Indications for $\mathrm{MeV}$ emission from the source is found only in some early Phases. Its time-averaged $\mathrm{MeV}$ spectrum does not allow to draw any conclusions.

The COMPTEL results have to be compared to those in additional energy bands, in particular to the neighboring $\gamma$-ray bands covered by EGRET and OSSE. This work is in progress and will be given by Zhang et al. [5].

ACKNOWLEDGMENTS: The COMPTEL project is supported by the German government through DARA grant 50 QV 9096 8, by NASA under contract NAS5-26645, and by the Netherlands Organization for Scientific Research (NWO).

\section{REFERENCES}

1. Hartman, R.C., et al., ApJ 407, L4 (1993).

2. Nolan, P.L., et al., $A p J$ 414, 82 (1993).

3. Blom, J.J., et al., $A \mathscr{B} A$ 295, 330 (1995).

4. McNaron-Brown, K., et al., ApJ 451, 575 (1995).

5. Zhang, S., et al., $A \mathscr{B} A$ in preparation (2001).

6. Schönfelder, V., et al., ApJS 86, 657 (1993).

7. Hartman, R.C., et al., ApJS 123, 79 (1999).

8. Lichti, G.G., et al., $A \& B A$ 298, 711 (1995).

9. Dermer, C.D., et al., ApJ 416, 458 (1993).

10. Collmar, W., et al., this proceedings (2001). 